University of Nebraska - Lincoln

DigitalCommons@University of Nebraska - Lincoln

Faculty Publications: Materials Research Materials Research Science and Engineering Science and Engineering Center

$1-1-2006$

\title{
Spin-Polarized Electronic Structure
}

\author{
Arti Kashyap \\ University of Nebraska-Lincoln, akashyap@Inmiit.ac.in \\ Renat F. Sabirianov \\ University of Nebraska - Omaha, rsabirianov@mail.unomaha.edu \\ Sitaram Jaswal \\ University of Nebraska, sjaswal1@unl.edu
}

Follow this and additional works at: https://digitalcommons.unl.edu/mrsecfacpubs

Part of the Materials Science and Engineering Commons

Kashyap, Arti; Sabirianov, Renat F.; and Jaswal, Sitaram, "Spin-Polarized Electronic Structure" (2006). Faculty Publications: Materials Research Science and Engineering Center. 29.

https://digitalcommons.unl.edu/mrsecfacpubs/29

This Article is brought to you for free and open access by the Materials Research Science and Engineering Center at DigitalCommons@University of Nebraska - Lincoln. It has been accepted for inclusion in Faculty Publications: Materials Research Science and Engineering Center by an authorized administrator of DigitalCommons@University of Nebraska - Lincoln. 
Published in Advanced Magnetic Nanostructures, edited by David J. Sellmyer and Ralph Skomski (Springer, 2006).

http://www.springer.com/east/home/generic/search/results?SGWID=5-40109-22-97856589-0

Copyright $(\odot 2006$ Springer Verlag. Used by permission.
Chapter 2

\section{SPIN-POLARIZED ELECTRONIC STRUCTURE}

\author{
A. Kashyap \\ IFW Dresden,Germany \\ R. Sabirianov \\ Department of Physics \\ University of Nebraska-Omaha \\ S. S. Jaswal \\ Department of Physics and Astronomy \\ University of Nebraska-Lincoln
}

Abstract

This chapter is devoted to the electronic structure of nanoscale metallic magnets. After an introduction to methods of electronic structure calculations, we review how recent trends translate into the description of magnetic nanostructures. Among the considered structures are nanowires, small particles, surfaces and interfaces, and multilayers, and emphasis is on magnetic properties such as moment and magnetization, interatomic exchange, and anisotropy.

\section{INTRODUCTION}

Nanostructures open new possibilities to tailor the mechanical, chemical, magnetic and electronic properties of materials and, at present, there is strong demand for basic understanding of new phenomena that nanostructures may exhibit. Nanomagnetic objects are different from both atoms and bulk materials, thereby providing an interface between physics, chemistry, material sciences, engineering and biology. For example, the length scale of typical nanostructures allows a direct use in many systems, including the human body. 
Some phenomena described by first principles are surface-and interface magnetic anisotropies, reduced or enhanced magnetic moments at particle or film surfaces, and optical properties, including magnetic dichroism. In addition, parameters obtained from first-principle calculations serve as an input in numerical micromagnetic simulations (Ch. 4).

Here we focus on iron-series transition-metal elements and their alloys. Magnetic oxides and rare-earth compounds can also be used in nanomagnets, but their magnetism goes beyond the scope of this review. For example, the large magnetocrystalline anisotropy of rare-earth transition-metal intermetallics, which are used in nanostructured permanent magnets, largely reflects the Hund's-rules ground state of tripositive rare-earth ions (Ch. 3 ).

Magnetic clusters containing a few atoms have been investigated for many decades, both theoretically and experimentally [1]. The electronic structure of atomic clusters was traditionally treated by real-space methods based on various approaches from tight-binding (TB) to density-functional methods $[2,3]$. The methods of calculations of electronic structure of bulk materials use periodic boundary condition and $\mathrm{k}$-space representation. They are very well developed and applied to broad variety of materials [4]. Nano-structures include 100-100000 atoms and do not exhibit periodicity, so that their first-principle study is a very challenging problem. Direct atomistic treatment of real nanodevices is very difficult due to the large number of atoms. The free-electron model, tight binding approaches and order $\mathrm{O}(\mathrm{N})$ density functional approach have all been applied to the nanoscale geometries to study their electronic properties. The choice of an approach depends on the degree of compromise between numerical and conceptual tractability on the one hand and quantitative accuracy on the other.

\section{METHODS OF ELECTRONIC-STRUCTURE CALCULATIONS}

Electronic structure methods for studies of nanostructures can be divided broadly into supercell methods and real-space methods. Supercell methods use standard k-space electronic structure techniques separating periodically repeated nanostructures by distances large enough to neglect their interactions. Direct space methods do not need to use periodic boundary conditions. Various electronic structure methods are developed and applied using both approaches. In this section we will shortly discuss few popular but powerful electronic structure methods: the pseudopotential method, linear muffintin orbital and related methods, and tight-binding methods.

\subsection{Linear Muffin-Tin Orbital and Related Methods}

Basis-set methods may be divided into two categories, depending on whether they (i) use wave function expansions in some set of fixed basis functions like atomic orbitals, Gaussian and plane waves, or (ii) expand the wave function in a set of energy and potential dependent partial waves as done in KorringaKohn-Rostoker (KKR) and the augmented plane wave (APW) methods. The KKR and APW methods require computational efforts which, despite attempts to improve the efficiency, are barely feasible and limited to sp-like valence and conduction electrons. Computationally, this can be remedied by the addition of localized orbitals to the plane wave basis set. But such a hybrid scheme is neither elegant nor in accordance with the chemical and physical intuition based on the smooth trends observed through the periodic table. It is necessary to use the self-consistent methods for computing one electron eigenvalues and eigenvectors with speed and accuracy. The so-called linear methods of band theory satisfy the requirements rather well. This is true for the linearmuffin-tin-orbital method (LMTO) [5-8]. This method is linearized version of the KKR method. Almost identical with the solid state LMTO method is the augmented spherical wave (ASW) method of Williams et al. [9].

The LMTO has also been extended to treating crystalline impurities with the Green's function technique by Koeing et al. [10]; and Gunnarson et al. [11] have used it for both metal and semiconductor hosts. Harris [12], Casula and Herman [13] and Springborg et al. [14, 15] have developed the LMTO method for clusters and molecules. For surfaces and thin films, LMTO techniques have been devised by Fuziwara [16] and Femando et al. [17]. It was also discovered that the conventional solid-state LMTO basis set can be transformed exactly into orthogonal [6,7], tight binding (TB) [6] and minimal [100] basis sets, which simplifies and generalizes the solid-state LMTO method considerably.

The use of the atomic-sphere approximation (ASA) [18], where the singleelectron potential is modeled as a superposition of spherical potentials inside overlapping space-filling spheres, makes LMTO methods computationally very efficient. Where this approximation is applicable, the LMTO-ASA method is presumably the most efficient procedure available for solving the density functional equations to a reasonably high degree of accuracy. However, a full-potential treatment going beyond the ASA is needed for many systems, including surfaces and impurities, and for total-energy changes associated with phenomena such as phonon distortions and atomic relaxations etc. A number of full potential methods have been developed, namely, FPLAPW $[19,20]$, FPLMTO [21] and FPLO [22]. A real-space version of the full-potential approach was implemented by Beck [23]. 


\subsection{Plane-Wave Pseudopotential Methods}

The pseudopotential method relies on the separation (in both energy and space) of electrons into core and valence electrons and implies that most physical and chemical properties of materials are determined by valence electrons in the interstitial region. One can therefore combine the full ionic potential with that of the core electrons to give an effective potential (called the pseudopotential), which acts on the valence electrons only. On top of this, one can also remove the rapid oscillations of the valence wavefunctions inside the core region such that the resulting wavefunction and potential are smooth.

Beyond a chosen cutoff radius, the all-electron and pseudofunctions (potential and wavefunction) are identical, while inside the core region both the pseudopotential and pseudowavefunction are smoothly varying. After the construction of these pseudofunctions for a single atom and ensuring that their scattering properties are almost identical to those of the all-electron functions, they can be used in any chemical environment.

The pseudopotential method has various advantages. Eliminating the core electrons from the problem reduces the number of particles that must be considered in the Kohn-Sham (KS) equations for the effective one-electron potential. For example, a pseudopotential calculation for bulk silicon (with 10 core and 4 valence electrons) requires the calculation of 4 occupied bands at each k-point, while an all-electron approach would require the calculation of 14 occupied bands. More importantly, the smooth spatial variation of the pseudopotential and pseudowavefunction allows the use of computationally convenient and unbiased basis, such as plane wave basis sets or grids in space.

The plane-wave pseudopotential method has proven to be an excellent computational tool for solving large scale electronic structure problems [24, 25]. Notable strengths of the method are the ability to use fast Fourier transform to update the Kohn-Sham equations, the lack of dependence on the basis on atom positions, and the clear control of convergence with the cutoff energy determined by the shortest wavelength mode. However, the method encounters difficulties in treating widely varying length scales. This issue is especially relevant for surfaces, clusters, and the hard pseudopotentials of first row elements or transition metals, which vary rapidly near the nucleus. A real-space version of the pseudopotential method was developed by Chelikowsky [26].

\subsection{Tight-Binding Methods}

The traditional approach to obtain the electronic structure of a periodic solid with $a b$ initio pseudopotentials has been to solve the Kohn-Sham (KS) equa- tions in momentum space using a plane-wave basis set. There are many advantages of plane-wave basis sets. For example, due to their completeness and orthonormality, they allow convenient and unbiased representation of the charge density and wavefunctions for calculating operator matrix elements. For non-periodic and localized systems, such as defects, surfaces, clusters, quantum dots, using a plane wave basis set requires an artificial periodicity in order to implement standard electronic structure algorithms. This artificial periodicity is introduced through the use of large unit cells called supercells.

While the supercell approach works well for localized systems, it is typically necessary to consider a very large supercell. This results in a plane-wave basis replicating not only the relevant electronic states but also vacuum regions imposed by the supercell. A much more efficient method to implement for investigating the electronic structures of localized systems is to use real space methods such as the recursion methods [27] and the moments methods [28]. These methods do not require symmetry and their cost grows linearly with the number of inequivalent atoms being considered. For these reasons, real space methods are very useful for a description of the electronic properties of complex systems, for which the usual k-space methods are either inapplicable or extremely costly.

Real-space methods are generally impractical, but they are extremely efficient when the system in consideration is well described by a tight-binding (TB) Hamiltonian. For a long time, parameterized TB Hamiltonians in conjunction with real space methods have been used to study the properties of complex transition metal alloys. In some cases, simple model TB Hamiltonians are very useful to stress the essential physics of the phenomenon in question and even to give the correct trends found in real systems. But in other cases, the effects are too subtle and the results obtained by the use of these parameterized Hamiltonians can be misleading. Even when trends can be well described, to obtain quantitative results a more exact Hamiltonians is usually needed. This has been a major problem regarding real-space electronic structure calculations. Usually, the involved parameters needed to build the TB Hamiltonian, such as hopping integrals, are obtained from a linear combination of atomic orbitals (LCAO) fit to more exact k-space calculations or are adjusted to fit experimental results [29].

When one considers complex systems, for which no exact results are available, parameters are usually obtained from a simple similar system and then transferred to the more complex system in question using the Slater-Koster rules [30] and some other empirical formulas which are known to be roughly obeyed. The results were often encouraging, but the lack of a solid theoretical background to justify the procedure left some fundamental questions unanswered. For example, how could one simulate the crystal-field splitting which 
depends on local environment and can not be transferred from one calculation to another, and how do we treat the wave function? The solution to these problems is not unique and several interesting points of view have developed. However, major progress towards obtaining a TB-Hamiltonian based on a solid theoretical understanding of the problem came in 1984 with the advent of the LMTO-ASA-TB formalism [31]. This gave rise to a new era regarding the quantitative description of the electronic structure of complex systems by using real-space methods

\section{MAGNETIC PROPERTIES}

In this chapter we describe how basic or "intrinsic" magnetic properties are calculated from first principles. Technological applications, for example in magnetic recording, tend to require a large magnetization, thermal stability, and control over the coercivity. The reversal process is described usually by micromagnetic modeling which takes into account dipole-dipole interactions. The description of magnetization reversal is beyond the scope of our paper. For details of micromagnetic modeling, readers are referred to Chs. 3 and 4 and to the specialized literature [31].

Magnetic properties can be understood and fully described from microscopic considerations using ab-initio electronic-structure methods. The local magnetic moment in combination with intra-atomic exchange defines the magnetization, interatomic exchange describes the thermal stability, and the coercivity reflects the magnetic anisotropy of the system. These interactions have different energy scales. The intra-atomic exchange is of the order of 2 $\mathrm{eV}$, interatomic exchange of the order of $10 \mathrm{meV}$, and anisotropy of the order of $1 \mathrm{meV}$. However, all of them are extremely important to describe the behavior of a magnet.

The description of nanomagnets requires new approaches. First, nanostructures are not periodic and tend to have large surface-to-volume ratios. Because of this the magnetization is not uniform across the nanostructure, local magnetic moments differ from site to site, exchange coupling varies throughout the nanostructure, and the anisotropy can be quite different from bulk or surface anisotropies. Second, it is hard to define properties in the similar fashion as in the bulk or as in case of molecules.

\subsection{Magnetic Moment}

The magnetic moment is given by the difference between the filling of majority and minority spin bands, which is directly related to the ferromagnetic exchange splitting between the bands. There is also an orbital-moment contribu- tion to the moment, but in 3d-based magnets, it is often small due to quenching (Ch. 3). Total energy minimization over all occupied band states yields crystal structure and magnetic ordering. Magnetic coupling and magnetic moment are sensitive functions of the interatomic spacing and the atomic volume, respectively. Both are dominated by the Coulomb and by exchange interactions at small distances. The moment is determined primarily by intraatomic exchange interactions and the Curie temperature by the interatomic exchange interactions.

The most common approach to calculate the magnetization from first principles is to use the local density formalism, where the spin dependent part of the total energy is approximated by an expression derived for the electron gas [32]. The corresponding exchange-correlation potential depends only on the majority and minority spin densities. Different magnetic structures, such as paramagnetic, ferromagnetic and various antiferromagnetic configurations, exhibit different total energies, and the lowest energy configuration obtained by local density theory represents the observed magnetic state in most cases. Room-temperature ferromagnetism occurs in rather few elements, $\mathrm{Fe}, \mathrm{Co}, \mathrm{Ni}$, $\mathrm{Gd}$, and several other rare earths are ferromagnetic at low temperatures. However, many alloys are ferromagnetic. The stability of the ferromagnetism of itinerant magnets can be explained by the Stoner criterion, which takes the DOS at the Fermi level, $D\left(E_{\mathrm{F}}\right)$, and an atomic exchange integral $I$ as input [33-35]. The transition from para-magnetism to ferromagnetism is favourable for $D\left(E_{\mathrm{F}}\right) I>1$. Then the system can lower its energy by bringing enough majority spin electrons down in energy by opening up the ferromagnetic exchange splitting. The Stoner criterion explains why Fe, Co and $\mathrm{Ni}$ are singled out for ferromagnetism. Several other elements are close to fulfilling the criterion, for example Pd. Since the DOS is different for different atomic sites, nanostructures may exhibit different local moments and complicated magnetic transitions.

With increasing atomic volume, one approaches the free atom limit where Hund's first rule postulates maximum spin, so that the individual spins of the electrons in a shell are aligned parallel. More generally, Pauli's exclusion principle implies that electrons with parallel spins have different spatial wavefunctions, reduces the Coulomb repulsion and is seen as exchange interaction. When the atoms are squeezed into a solid, some of the electrons are forced into common spatial wavefunctions, with antiparallel spins and reduction of the overall magnetic moment. At surfaces and interfaces, the reduced coordination reverses this effect, and a part of the atomic moment is recovered. 


\subsection{Anisotropy}

Magnetic anisotropy is one of the most important properties of a magnetic material. As mentioned in Ch. 1, it is the energy necessary to deflect the magnetic moment in a single crystal from the easy to a hard direction. The main contribution to the anisotropy is magnetocrystalline anisotropy. The easy and hard directions arise from the interaction of the spin magnetic moment with the crystal lattice that is due to spin-orbit coupling. In crystals, the magnetocrystalline anisotropy energy is given by a series expansion in terms of the angles between the direction of magnetization and the crystal axes (Ch. 4). It is often sufficient to represent the anisotropy energy in an arbitrary direction by just the first two terms in the series expansion. These two terms each have an empirical constant associated with them called the first- and second order uniaxial anisotropy constants $\mathrm{K}_{1}$ and $\mathrm{K}_{2}$ respectively. In cubic crystals, the lowest-order term is the fourth-order term, also known as the "cubic" $\mathrm{K}_{1}$.

There is also some shape anisotropy due to magnetostatic dipole interactions (Ch. 3). This anisotropy is important in some nanomagnets, for example in elongated nanoparticles, but unrelated to the electronic structure.

A contribution caused by spin-orbit coupling and closely related to magnetocrystalline anisotropy is magnetoelastic anisotropy. Mechanical stress creates a strain which amounts to a lattice distortion and yields a correction to the magnetocrystalline anisotropy. Surface anisotropy is a manifestation of magnetocrystalline anisotropy, too (sections below and $\mathrm{Ch}$. 3).

Starting with Brooks (1940), calculations of the magnetocrystalline anisotropy energy (MAE) of itinerant magnets have developed in two different approaches have been developed since the pioneering studies of Takayama, Bohnen, and Fulde [36] and of Gay and Richter [37]. The first approach uses perturbation theory within a semi-empirical tight-binding framework, justified by the argument that the MAE is small compared to a characteristic bandwidth $[36,38,39,40]$. The second approach relies on ab initio spin-polarized total-energy calculations, with spin-orbit coupling included either self-consistently within the scalar-relativistic approximation [41, 42] or as a final perturbation to a calculation neglecting spin-orbit coupling [37, 43-45] and using the force theorem $[46,47]$. The main difficulty with this approach arises from the fact that a change in the direction of the magnetic moment changes the occupation of the eigenstates only in a narrow region of the Brillouin zone, hence the $k$-space integrations for the total energy are only very slowly convergent. To avoid the necessity to use a huge number of $k$-points, Wang, Wu, and Freeman [45] introduced a state tracking procedure, using information on the change of the band structure with increasing spin-orbit interaction to extrapolate the Brillouin-zone integrals.
The description of the magnetic anisotropy of nanoparticles is more complicated than that of bulk systems, partially because shape and surface anisotropies (Ch. 3) are of the same order of magnitude as the magnetocrystalline anisotropy. The ratio of atoms on the surface to the number of atoms in the core of a nanoparticle is very large. This changes the electronic structure of the core atoms as well and its anisotropy may be quite different from the bulk systems. Surface anisotropy may cause drastic change in magnetization and reverse properties of nanostructures. Even in a naive magnetostatic approach, surface anisotropy causes the renormalization of switching parameters [48].

\subsection{Spin Structure and Noncollinearity}

The term spin structure refers to the ferromagnetic, antiferromagnetic or ferrimagnetic alignment of the atomic spins. In ferromagnets, all spins are parallel and the atomic moments add, whereas ferromagnets and antiferromagnets are characterized by two or more sublattices with opposite moments. This leads to a reduction or absence of a net magnetic moment. Competing exchange interactions in periodic crystals and in disordered magnets give rise to noncollinear spin arrangements. Examples are helimagnetic order in perfect crystals, which is caused by the competing interactions between next and more distant neighbors, and spin-glass behaviour in magnets with atomic scale disorder [49]. Deviations from parallel or antiparallel spin alignment may also occur at surfaces and interfaces. Further, it is important to distinguish between zero temperature magnetism and finite temperature magnetic order.

It is difficult to calculate finite-temperature magnetic properties from first principles, particularly Curie and Neel temperatures. In the atomic limit, for example using the Heisenberg model, one assumes a magnetic moment on each atom that persists beyond Curie temperature. In the paramagnetic phase, the magnetization vanishes by orienting the moments at random. The other extreme, that is the Stoner model of band like ferromagnetism, assumes that the magnetic moment gradually decreases at elevated temperature and vanishes at the Curie temperature. It yields Curie temperatures that are several times higher than observed [35]. The basic drawback is the assumption that the magnetic order disappears by spin flip excitations across the Stoner gap. They cost more energy than is actually needed to disorder magnetic moments. More realistic models contain small domains with local order whose orientations are starting to fluctuate when approaching the Curie temperature $(\mathrm{Ch}$. 3). Some degree of local correlation remains even above $T_{C}$. Such a picture brings in spin wave excitations (magnons) which are not contained in the ground state energy bands. The efforts to model the spin structure just below and above the Curie point from first principles give qualitatively correct Curie 
temperatures [50], even if the method used in the paper is more appropriate to very weak itinerant ferromagnets such as $\mathrm{ZrZn}_{2}$ (Ch. 3)

\subsection{Interatomic Exchange}

Magnetic order occurs because the magnetic moments of neighbouring atoms couple by interatomic exchange. As the intra-atomic exchange responsible for the magnetic moment, it reflects the overlap of electrons in combination with Pauli's exclusion principle. Depending on the positive or negative sign of the exchange, the interaction between the ions will force the individual moments into parallel (ferromagnetic) or antiparallel (antiferromagnetic) alignment. The direct exchange between overlapping orbitals was proposed first. Later other types of interatomic exchange were identified such as an indirect or RKKY-type exchange, superexchange, double exchange, anisotropic exchange, and higher-order exchange (such as biquadratic). All of them may be present in a system and responsible for a variety of effects governing the magnetic structure and finite-temperature behaviour of magnet.

Direct exchange operates between moments which are close enough to have sufficient overlap of their wavefunctions. it gives a strong but short range coupling which decreases rapidly as the ions are separated. Direct exchange as originally envisaged by Heisenberg, is always ferromagnetic, but there is a negative hopping contribution. When the atoms are very close together, the electrons minimize the single-electron interaction by spending a considerable time between the nuclei. Since the electrons are then at the same place in space at the same time, Pauli's exclusion principle requires them to possess opposite spins. This gives rise to antiparallel alignment, corresponding and therefore negative or antiferromagnetic exchange. Note that the direct intra-atomic exchange between electrons in the same atom $\mathrm{J}$ is positive, leading to Hund's first rule.

Indirect exchange couples moments over relatively large distances. It is the dominant exchange interaction in metals where there is little or no direct overlap between neighbouring magnetic electrons. It therefore acts through an intermediary which in metals is the conduction electrons (itinerant electrons) This type of exchange is also known as the Ruderman, Kittel, Kasuya and Yoshida (RKKY) interaction, especially when mediated by free electrons. This spin polarization in the itinerant electrons is felt by the moments of neighboring magnetic ions within range, leading to an indirect coupling. Since RKKY exchange oscillates from positive to negative as the separation of the ion changes, the magnetic coupling can be ferromagnetic or antiferromagnetic. In rare-earth metals whose magnetic electrons in the $4 \mathrm{f}$ shell are shielded by the $5 \mathrm{~s}$ and $5 \mathrm{p}$ electrons, direct exchange is rather weak and insignificant and in- direct exchange via the conduction electrons gives rise to magnetic order in these materials.

A method to calculate $\mathrm{J}_{\mathrm{ij}}$, based on the local approximation to spin density functional theory has been developed by Liechtenstein et al. [51, 52]. Using spherical charge and spin densities and a local force theorem, expression for $\mathrm{J}_{\mathrm{ij}}$ is

$$
J_{i j}=\frac{1}{4 \pi} \sum_{L L^{\prime}} \operatorname{Im} \int_{-\infty}^{\varepsilon_{F}} d \varepsilon \Delta_{l}^{i}(\varepsilon) T_{L L^{\prime}}^{i \uparrow}(\varepsilon) \Delta_{l^{\prime}}^{j}(\varepsilon) T_{L L^{\prime}}^{i \downarrow}(\varepsilon)
$$

Here $T_{L L^{\prime}}^{i j}(\varepsilon)$ is the scattering path operator in the site $(\mathrm{i}, \mathrm{j})$ representation for different spin projections $(\sigma=\uparrow, \downarrow)$, and $\Delta_{l}^{i}(\varepsilon)=t_{i \uparrow}^{-1}-t_{i \downarrow}^{-1}$ is the difference of the inverse single-site scattering matrices. The total exchange of a given site with all sites $\left(J_{o}=\sum_{i \neq 0} J_{o i}\right)$ can also be calculated from the relation

$$
J_{0}=\frac{1}{4 \pi} \sum_{L L^{\prime}} \operatorname{Im} \int_{-\infty}^{\varepsilon_{F}} d \varepsilon\left\{\Delta_{l}^{0}(\varepsilon)\left[T_{L L^{\prime}}^{00 \uparrow}(\varepsilon)-T_{L^{\prime}}^{00 \downarrow}(\varepsilon)\right]+\Delta_{l}^{0}(\varepsilon) T_{L^{\prime}}^{\infty \uparrow} \Delta_{l^{\prime}}^{0}(\varepsilon) T_{L L^{\prime}}^{00 \downarrow}(\varepsilon)\right\}
$$

The parameter $J_{0}$ reflects the energy change due to small-angle rotation of the moment at one site. In contrast to the $J_{\mathrm{ij}}$, it is given by the site-diagonal scattering matrix (or Green function), where $i=j$. This approach has been used to study the magnetism of Y-Fe, Y-Co and $L 1_{0}$ systems [53-55].

\section{ELECTRONIC STRUCTURE OF NANOMAGNETS}

Recent advantages in the preparation and characterization of thin films, surfaces and nanostructures have lead to an intriguing question: is it possible to fabricate 'designer solids' by controlling materials on the atomic scale, that is layer by layer, row by row, and ultimately atom by atom? Engineered molecules are common place in biochemistry, and the same idea can be apply to solids and electronic materials. Electronic properties of semiconductor devices have been controlled by heterostructures, quantum wells and superlattices. Magnetism as a cooperative phenomenon also lends itself to manipulation in small structures, where neighboring atoms can be replaced systematically by species with stronger or weaker magnetism. In fact, a class of magnetic/nonmagnetic multilayers termed "spin valves" has been introduced into magnetic storage devices (Ch. 14). The wave functions of electrons undergo changes on confining them to dimensions comparable with their wavelength. In semiconductors, confinement and quantization phenomena are visible already at dimensions greater than $200 \mathrm{~nm}[56,57]$, whereas in metals they typically are 
seen at $1 \mathrm{~nm}$. In fact, the Fermi wavelength of typical metals has atomic dimensions, but beat frequencies with the lattice can be an order of magnitude larger.

Consider the formation of low dimensional electronic states by quantization. Confining electrons to small structures causes the continuous bulk bands to split up into discrete levels, for example quantum well states in a slab. For $N$ atomic layers in the slab there are $N$ levels. In order to exhibit two dimensional behavior there should be only a single level within about 6 $k_{\mathrm{B}} T$ of the Fermi level. Several levels within the Fermi cutoff would already approach a three dimensional continuum. For a coarse estimate of the corresponding slab thickness, one may set the energy $E$ of the lowest level equal to $k_{\mathrm{B}} T$. For room temperature $\left(E=k_{\mathrm{B}} T=0.026 \mathrm{eV}\right)$, one obtains a de Broglie wavelength $h / p=h /(2 m E)^{1 / 2} \sim 8 \mathrm{~nm}$, which is comparable with the spatial extent of the lowest quantum state. Thus, both the high electron density in magnetic metals and the requirement of room temperature operation for quantum devices point to dimensions of a few nanometers. Note, however, that Fermions are quantum-mechanical objects at any temperature below $E_{\mathrm{F}} / k_{\mathrm{B}}$.

The contribution of first-principle calculations to physics, chemistry, materials science, and, recently, geology and biology is more important than ever. The main reasons are the steady increase in computer power and the continuous progress in methodology (both efficiency and accuracy of algorithms and approximations). However, the application of these methods to investigate structural, electronic, and optical properties of nanostructures has not been straightforward due to extensive computational needs and to the new physics inherent in the nanometer and subnanometer region. One particularly useful extension to overcome the computational demand imposed by localized nanostructures has been the introduction of methods based on a real-space implementation, such as the higher-order finite-difference pseudopotential methods. In the most favorable cases, quantum mechanical formulations of the electronic structure of atomic systems scale as the cube of the number of atoms of the system. This makes it very difficult to reach system sizes larger than a few hundreds of atoms, and is therefore a huge barrier for the study of problems in nanoscale materials. The way out of this trap was found several years ago, when a number of ideas suggested the possibility of developing approximate although accurate schemes to reduce the computational cost to linear scaling. These so called $O(N)$ [58] methods have matured since those first proposals, and now constitute a viable route for studying systems with unprecedented size.

\section{FIRST-PRINCIPLE STUDIES OF NANOSTRUCTURES}

Recent advances in theoretical methods and high-performance computing allow for reliable first principle calculations of complex nanostructures. Nanostructured materials are characterized by a fascinating diversity of geometries, but here we restrict ourselves mainly to first-principle calculations for nanoparticles and clusters, nanowires and nanocontacts. Nanoscale multilayers are also discussed very briefly, although multilayers are often considered as a subfield of thin-film physics rather than nanoscience. We also ignore nanotubes, because most of the work in this direction has been done on nonmagnetic carbon nanotubes.

\subsection{Nanoparticles and Clusters}

In magnetic recording, a bit of information corresponds to a small area or volume of ferromagnetic material with a well-defined and as high as possible magnetic moment. Furthermore, below a given size the grains tend to become superparamagnetic at room temperature, and the stored information would become unstable. It is known, both experimentally and theoretically, that the magnetic moments and the magnetic anisotropy energy per atom are often enhanced in small metallic clusters, as compared to the bulk crystalline state. Nanosize transition-metal particles, between the molecular and metallic states, are of particular interest in this context due to their intrinsically high magnetic moment. The properties of these clusters depend strongly on its surrounding. We will compare magnetic properties of free-standing clusters, clusters supported on substrate, and clusters embedded in the media.

Studies of small free-standing FeN, CoN and NiN clusters have revealed that their magnetic moments are significantly larger than the corresponding bulk magnetizations [59-65]. Non-vanishing magnetic moments have even been observed in small clusters of some $4 \mathrm{~d}$ transition metals, for example $\mathrm{Pd}$, $\mathrm{Ru}$ and $\mathrm{Rh}$, which are non-magnetic in the solid state [66-70]. For example, Kumar et al. performed ab initio calculations on the growth and magnetic behavior of Pd clusters having up to 147 atoms and found an icosahedral growth and a ferromagnetic behavior in these clusters [68]

The magnetization of free clusters of elemental metals exhibits an oscillatory behavior with increasing cluster size. The origin of these oscillations is the successive addition of atomic shells with increasing cluster size. The variation of the magnetic moment in the outermost shell is due to the changing coordination number of surface atoms. As a rule, surface atoms with lower coordination (lower number of nearest neighbors) have larger moments, and the corresponding theory of magic numbers seems to explain experimental observations. 
Using a tight-binding Hubbard Hamiltonian in the unrestricted Hartree-Fock approximation, Pastor et al. [71] have shown the size and structural dependence of magnetic properties of free $\mathrm{Cr}_{\mathrm{n}}, \mathrm{Fe}_{\mathrm{n}}$, and $\mathrm{Ni}_{\mathrm{n}}(\mathrm{n}<15)$ clusters, and also the exchange interaction and local environments effects in $\mathrm{Fe}_{\mathrm{n}}$ clusters [72]. The finite-temperature magnetism of small clusters, which is remarkably different from that of bulk systems, has also been studied in terms of a similar approach by taking into account both electronic and structural excitations [73]. A great advantage of the TB methods is that they easily can be combined with molecular dynamics calculations, enabling the investigation of relaxation effects which proved to be important in determining the magnetic moments and the MAE of transition metal clusters [74-76]

As mentioned above, the anisotropy of the free standing clusters is very complicated. Experimental studies predict a very complicated switching behavior, suggesting a strong competition between different contributions to the anisotropy energy $[77,78]$. The main reason is that different pairs of spin-orbit split levels yield anisotropy contributions of opposite sign, so that the net anisotropy is the sum of many positive and negative contributions. Phenomenological studies complement this point and yield good agreement with experiment $[79,80]$.

The supercell-based studies of supported clusters show that the magnetic moment tends to increase as well [81]. For example, small Co nanoclusters may exhibit a large orbital moment and large anisotropy on Pt surface [82]. The pros and cons of large orbital moments in applications of nanostructures was discussed by Andriotis et al. [83]. Magnetic-nanoparticle patterned media are extensively studied as a potential breakthrough technology for highdensity storage of information. The key requirement is that the individual nanoparticles exhibit both a large magnetic moment and a high anisotropy. More generally, in the last decades, theoretical and experimental studies have shown that the reduction of size and dimensionality can give rise to a large range of novel materials. This has opened the possibility of generating specifically designed nanomaterials with tailored properties. Understanding the conditions for the development of such properties is crucial in order to be able to properly adjust and control these parameters, in particular for magnetic applications.

The embedding technique based on the Korringa-Kohn-Rostoker (KKR) Green-function method in the local spin-density approximation (LSDA) has been applied to the magnetism of transition metal adatoms and clusters deposited on surfaces. The main feature of this approach is that the interaction between adatoms and host surface atoms can be analyzed within first-principles electronic structure calculations $[84,85]$. An accurate calculation of the total energy in terms of full potential or full charge density schemes made possible the investigations of the energetics of adatoms [86-88]. As compared to
TB methods, an obvious drawback of the embedded KKR technique is that with respect to computational limitations, the number of the atoms in the cluster is restricted to about less than 100. Furthermore, the inclusion of structural relaxations is exceedingly difficult. On the level of a fully relativistic spinpolarized electron theory, recently, strongly enhanced orbital magnetism and MAE of adatoms and small clusters on Ag and Au (100) surfaces have been reported $[89,90]$.

Spin polarized electronic structure calculations of the Co clusters embedded in $\mathrm{Cu}$ matrix have been reported by Qiang et al. [91]. The authors found that the magnetization of the embedded Co clusters in $\mathrm{Cu}$ matrix is somewhat lower than the magnetization of the bulk Co, i.e. the effect is opposite to the free cluster case. This is caused by the slight suppression of the moment of cobalt sites in the interface region. The magnetization oscillations are similar to the free case in theory if shell by shell filling of the clusters is expected. However, this condition is not satisfied in case of embedded clusters [92] and magic number theory does not work. Moreover, there is always some interdiffusion on the interface between atoms of the matrix and atoms of the cluster in case of larger clusters. Frequently it creates a magnetically dead layer at the interface.

The exchange interactions between metallic clusters in metallic media exhibit oscillatory dependence on the intercluster distance like it happens in Co clusters embedded in the $\mathrm{Cu}$ matrix (see also $\mathrm{Ch}$. 3). The calculated intercluster exchange interactions shows both ferromagnetic and aniferromagnetic exchange between clusters, and the values of exchange at short distances is of the order of interatomic exchange in the bulk cobalt. The spin-glass type behavior in this and similar systems arises from frustration. This is very likely responsible for the lowering of the magnetization with the increase of cobalt concentration from 10 to $50 \%$ [91].

Nanostructured permanent magnets use the idea of mixing soft magnetic materials and hard magnetic materials in a composite structure. The goal is to combine a large magnetocrystalline anisotropy of hard magnetic materials and large magnetization of the soft magnetic materials in order to obtain large energy product $(\mathrm{BH})_{\max }$, which requires large magnetization and large coercivity. This idea was first proposed by Kneller and Hawig [93], by estimating the effect in multilayers. Micromagnetic calculations based on parameters obtained from first principles show that $\mathrm{FePt} / \mathrm{Fe}$ nanostructures may exhibit a $(B H)_{\max }$ as high as 90 MGOe [54]. These changes are experimentally observed in various systems [94], and Section 2.3 in the following chapter discusses some systems.

The properties of embedded nanoclusters can be controlled by the properties of the matrix. For example, the matrix can exert uniaxial or biaxial strain on the embedded nanoclusters. The corresponding strain can modify the prop- 
erties of nanostructure considerably. For example, Zeng et al. show that applied stress can modify the Curie temperature and anisotropy of FePt [95].

\subsection{Nanowires}

The progress in experimental techniques has made it possible to synthesize stable ultrathin metal nanowires with diameter down to atomic sizes and of high aspect ratios [96-99]. An example is single-crystalline silver nanowires having a width of $0.4 \mathrm{~nm}$ width and deposited into the pores of an organic template [99]. Kondo et al. [100] have observed novel helical multishell structures in the suspended ultrathin gold and platinum nanowires. This is a relatively mature field of studies with thousands of articles published in the last few years, and there are various review articles available [101, 102]

While magnetic properties of free-standing nanoclusters have been extensively studied experimentally, studies of magnetic nanowires are largely limited to theoretical calculations. One exception are wires freed from templates, where mechanical stability excludes very thin diameters. Decades ago, Weinert and Freeman [103] investigated the electronic structures and magnetism of the linear $\mathrm{Fe}$ and Ni chains by using the self-consistent full-potential linearized augmented plane wave (FLAPW) method. They predicted large magnetic moments of $3.3 \mu_{\mathrm{B}}$ and $1.1 \mu_{\mathrm{B}}$ for the $\mathrm{Fe}$ and the Ni chains, respectively. Free-standing Co atomic chains show an enhanced magnetization of $2.3 \mu_{\mathrm{B}}$ as well. [104]

It is interesting that first-principle calculations predict ferromagnetism in monatomic nanowires of the nonmagnetic transition metals $\mathrm{Ru}, \mathrm{Rh}$, and $\mathrm{Pd}$, with respective mean-field moments of $1.1,0.3$, and $0.7 \mu_{\mathrm{B}}$ at the equilibrium bond length [105]. An analysis of the band structures indicates that the nanocontact superparamagnetic state suggested by calculations should affect the ballistic conductance between tips made of $\mathrm{Ru}, \mathrm{Rh}$ or $\mathrm{Pd}$, leading to possible temperature and magnetic-field dependent conductance. One method to produce nanowires is the growth on the steps of the crystalline surface cut with the high Miller index [106]. Spisak and Hafner [107] investigated the position dependent magnetic moments of the Fe nanowire on a vicinal $\mathrm{Cu}(117)$ by using the pseudopotential method. From the total energy comparison, they found that the system of the Fe nanowire placed on the inner comer site of the $\mathrm{Cu}(117)$ step is most stable with a magnetic moment of $2.80 \mu_{\mathrm{B}} /$ atom, while the system of the Fe nanowire placed on the top of the terrace (formed by (001) plane) of $\mathrm{Cu}(117)$ is unstable with a magnetic moment of $2.96 \mu_{\mathrm{B}} /$ atom. Jin et al. [108] investigated the electronic structures and magnetism of an Fe nanowire along the [010] direction on a $\mathrm{Cu}(001)$ surface (Fe [010] chain) by using the FLAPW method and found large magnetic moments of the Fe atoms: 3.11 and $3.39 \mu_{\mathrm{B}} /$ atom for the $\mathrm{Fe}[010]$ chain and the free-standing nanowires
(free-Fe[010] chain), respectively. Since free-standing nanowires of elemental metals are one-dimensional objects, they exhibit magnetic properties similar to free standing clusters, i.e. its magnetic moment increases especially for the atoms at surface. The lower coordinated atoms have higher moment, while atoms inside of the nanostructure have magnetic moment close to the bulk values.

Recently, investigations of the electronic structures and magnetism of an Fe chain along the [110] direction on a $\mathrm{Cu}(001)$ were performed by using the FLAPW method within the generalized gradient approximation [109]. Hong et al. [104] have calculated properties of $\mathrm{Co}$ atomic wires on the $\mathrm{Cu}(001)$ surface. They show that magnetic moment of atomic size wires can be affected by the substrate. The magnetocrystalline anisotropy of $\mathrm{Co}$ wires supported on $\mathrm{Cu}$ substrate has opposite sign from the free standing case, i.e. the magnetization is along the wire on the $\mathrm{Cu}$ surface and perpendicular in free-standing case. Gambardella et al. [110] investigated a quasi-one-dimensional Co chain at the $\mathrm{Pt}(111)$ step edge. The symmetry breaking at the step leads to an easy magnetization axis at an odd angle of about 20 degrees towards the Pt step. The orbital and spin moment may have different orientation in this system. This reflects the specific character of the crystal-field interaction in these systems.

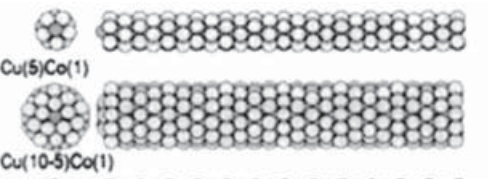

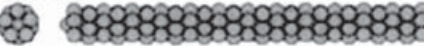

\section{$\cos$}

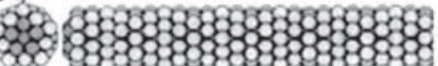

cul(10) $\cos (5-1)$

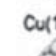

cut $10 \mathrm{cos}$
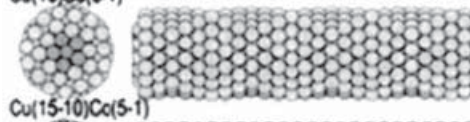

cul15-10) Co(s. -1)

$\cos (0.5)$

8308

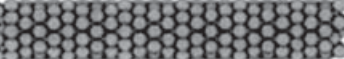

cu(is) Co(16-s-1)
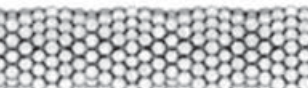

Figure 1. Optimized structures of bare and $\mathrm{Cu}$-coated Co nanowires (dark balls: Co atoms; bright balls, $\mathrm{Cu}$ atoms). Left part is for the centered hexagonal multishell structural pattern and right one for the centered pentagonal one. The indexes of n-nl-n2-n3 in parenthesis are used to characterize each structure [111].
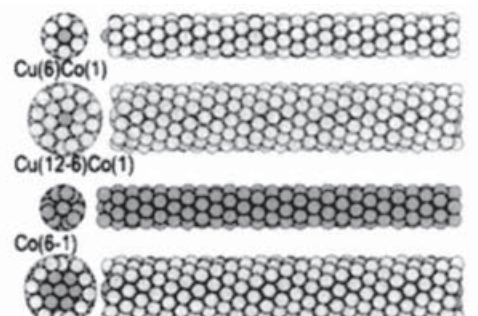

cu(12) $\operatorname{col}(6-1)$

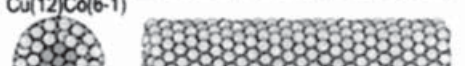

Cu(11.12) $\cos (6-1)$
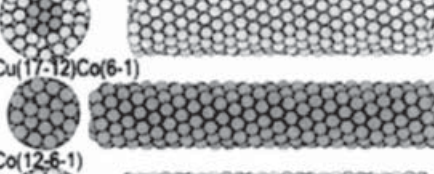

(3)

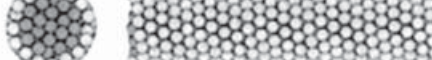


It is difficult to tackle the problem of embedded nanowires. An example is $\mathrm{Co}$ nanowires fully coated with atomic $\mathrm{Cu}$ shells. Wang et al. [111] find that $\mathrm{Cu}$ atoms occupy the surface, while Co atoms prefer to occupy the interior of the nanowires to form the perfect-coated structures. The coated $\mathrm{Cu}$ atomic layer leads to a large variation of the magnetic moment of the Co nanowires, depending on the structure and the thickness of $\mathrm{Cu}$ layers. A single row of $\mathrm{Co}$ atoms in the center of nanowire is found to be nonmagnetic when coated with two $\mathrm{Cu}$ layers, while all other investigated Co nanowires (Fig. 1) remain magnetic, albeit with reduced magnetic moments.

The transport properties of nanowires are of technological importance and have attracted significant attention in the recent years. Band structure gives simple solution to the analysis of the ballistic transport of periodic nanowires because the number of the bands crossing the Fermi surface is equal the number of quantum of conductance. However, the situation in nanocontacts is more complicated [112].

\subsection{Nanocontacts}

The understanding of spin transport in order to use it in electronics devices has been a field of intense research for the past few years. Interesting effects were reported in electrodeposited Ni nanocontacts, where resistance changes by a huge value on application of a small magnetic field at room temperature $[113,114]$. This has followed after effort was focused on the magnetoresistance effect of electrodeposited nanocontacts [115-117]. Smaller magnetoresistance ratios, but interesting from the point of view of applications, were reported also for $\mathrm{Co}, \mathrm{Fe}$ and $\mathrm{Fe}_{3} \mathrm{O}_{4}$ nanocontacts [118].

There have been several and partially exclusive attempts to explain the experimental data. One possibility considered is the scattering of the electrons in a domain wall [117-119]. The width of a domain wall in a nanocontact is predicted to be of the order of the nanocontact size [120] and such a reduction of the domain wall width is likely to enhance the magnetoresistance. However, this effect is very small, unless the domain walls contain atomically sharp feature, especially layers with reduced exchange [102]. Magnetostriction effects could also lead to an increased magnetoresistance [118], but most probably cannot explain the experiments. Alternatively, a thin domain wall pinned in a magnetic dead layer together with the possibility of $100 \%$ polarization of the Ni d electrons was also proposed as a possible explanation [114, 115]. Using a free-electron model and a linear response theory Zhuravlev et al. [121] investigated a spin-dependent electronic transport in magnetic nanocontacts in the ballistic regime of conduction. In particular, they show that the presence of a nonmagnetic region in the nanocontact separating two ferromagnetic elec- trodes can lead to a spin blockade resulting in very large values of magnetoresistance. Other possible explanations are a large magnetostriction in the experimental samples, or the presence of the defects in the constriction. The latter may result in the resonant tunneling and provide large MR.

In order to describe the nanocontacts theoretically, several methods have been developed by various groups. Recently, Burton et al. [122] investigated the electrical resistance of a constrained DW in a nanocontact using micromagnetic modeling and ballistic conductance calculations. They found that for electrodes with significantly different magnetic anisotropy, the DW can be trapped by the nanoconstriction (domain-wall pinning). They showed that the DW width and, therefore, the conductance can be controlled by an applied magnetic field. The first-principle approach is based on the tight-binding (TB) formulation [123-125]. An important conclusion coming from TB models is that conductance of the single-atomic contacts is proportional to the number of valence orbitals available at the Fermi energy $[124,126]$. Ab-initio density functional (DF) calculations with jellium electrodes have been proposed by Lang et al. [127-130] and Kobayashi et al. [131-133], which addressed single-atom contacts [128,134], atomic chains of $\mathrm{Al}[127,131], \mathrm{Na}[128,133]$, and $\mathrm{C}[130]$.

The formation mechanisms of atomic chains made from different types of elements such as $\mathrm{Ni}, \mathrm{Pd}, \mathrm{Pt}, \mathrm{Cu}, \mathrm{Ag}$ and $\mathrm{Au}$ were studied by means of molecular dynamic simulations [135]. Also, Mehrez et al. [136] and Brandbyge et al. [137] presented fully self-consistent DF calculations of the conductance of atomic contacts treating the electronic structure of the whole system (electrodes and the constriction region) on the same footing. The ballistic conductance through $\mathrm{Ni}, \mathrm{Co}$, and $\mathrm{Fe}$ nanocontacts within a semiempirical tight-binding model proposed by Velev and Butler [138] shows that the interplay of the contact and the domain-wall resistance can produce very large giant magnetoresistance ratios. However, the very giant magnetoresistance is limited to geometries in which the nanocontacts are very narrow and have very small aspect ratios, similar to the predictions in [102]. Solanki et al. [112] calculated the electronic structure and conductance of atomic-size Ni contacts using a real-space tight-binding LMTO method and recursion technique within the frame work of density functional theory. They used the Landauer-Buttiker approach to calculate the conductance. Their results of spin-dependent conductance as a function of energy show ballistic bulk-like behavior. The appreciable dependence of the conductance on the structure of nanocontact is reported in their work. Figure 2 shows an example of nanocontact modeling. Similar conclusion was made by Emberly et al. considering carbon nanowires [139].

We should notice that there are several research groups which could not reproduce giant MR in the nanocontacts experimentally. 


\subsection{Multilayers, Surface, and Interfaces}

Magnetic thin films and multilayers have attracted much interest in the context of nanomagnetism, although they are often considered as a separate branch of condensed matter physics [140]. Ultrathin magnetic films, epitaxially grown on nonmagnetic substrates, are prototype systems for investigating magnetism in two dimensions, and the possibility to produce films and multilayers with perpendicular magnetic anisotropy for magnetooptic recording applications is of technological importance [141, 142]. The topic of perpendicular magnetic anisotropy is very challenging, since the magnetostatic dipolar interactions always prefer in-plane orientation of the easy axis of magnetization. Since the pioneering experimental work by Gradmann [143] and the theoretical predictions of Gay and Richter [38] much effort has been spent investigating $\mathrm{Fe}, \mathrm{Co}$, and Ni films grown on noble-metals substrates. Among the heteroepitaxial systems that show strong perpendicular magnetic anisotropy (PMA) are $\mathrm{Co} / \mathrm{Au}(111)$ thin films and superlattices [144], Ni/Cu(001) [145-147] and $\mathrm{Ni} / \mathrm{Cu}(111)$ [146] thin films, $\mathrm{Co} / \mathrm{Au}(111)$ thin films and multilayers [144, 148], bcc $\mathrm{Fe} / \mathrm{Ag}(001)[149,150]$ and fcc $\mathrm{Fe} / \mathrm{Cu}(001)[151,152]$ thin films. Sabirianov and Jaswal carried out first-principles studies of exchange-coupled hard-soft multilayers predicting very large energy products needed for hardmagnet applications [153].

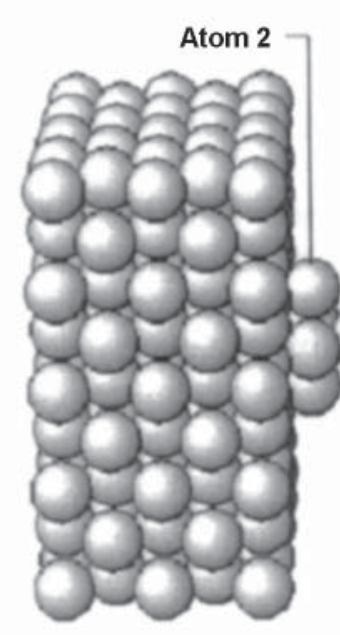

Left Lead

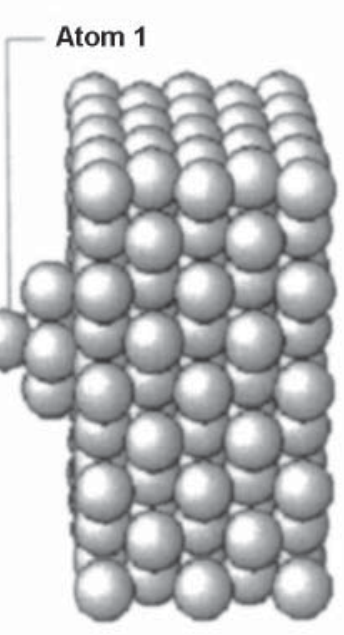

Right Lead
Figure 2. Geometry of a Ni nanocontact used in calculations. Atom of type 1 and atoms of type 2 occupy two non-equivalent sites within the nanocontact region [112].
The discovery of the giant-magnetoresistance (GMR) effect [154] has further renewed interest in the theory of transport in layered and granular systems. Several approaches to transport in layered systems are currently being used. The phenomenon of GMR is promising for its applications, such as in nonvolatile magnetic random-access memories in the information technology industry or as reading heads and various kinds of sensors in the recording and car industries, respectively. The GMR effect has been observed mostly in the diffusive transport regime in which the mean free path is much smaller than the dimension of the so-called active part of the multilayer system. In the ballistic regime, the mean free path is larger than the dimension of the active part of the multilayer system. Transport in multilayers has been studied extensively and review on the subject [155] covers the topic thoroughly.

The magnetocrystalline anisotropy of multilayered systems has been also calculated by many groups [156-158]. Újfalussy et al. [159] performed fully relativistic spin-polarized local spin density calculations for Co monolayer on $\mathrm{Au}(111)$. They obtained an enhancement of perpendicular magnetic anisotropy as a function of the Au coverage. They showed the close relationship between the anisotropies of orbital magnetic moments and the anisotropy energies, thus interpreting their results in terms of familiar perturbation theory. Lorentz and Hafner [157] presented calculations of the magnetic structure and of the uniaxial and planar anisotropies of thin films of $\mathrm{Fe}$ on $\mathrm{Cu}(001)$ substrates. They have used the real space recursion calculations using TB-LMTO to calculate the anisotropy. Their results for Fe monolayers reveal the importance of the surface and interface anisotropies, including the effect of nonmagnetic coverages. Essentially, this is a crystal-field effect, similar to that in bulk

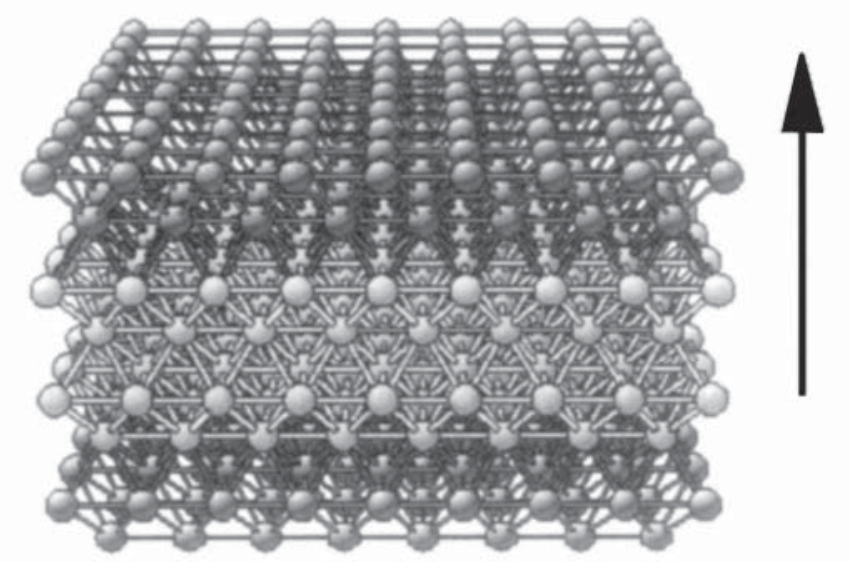

fcc (00I)

Figure 3. The modeling of layered structures for the calculational investigation [160]. 
compounds consisting of atomic layers with different magnetic properties. Stepanyuk and Hergert have [160] performed molecular dynamics simulations of $\mathrm{Co}$ nanostructures on $\mathrm{Cu}(001)$ and $\mathrm{Cu}(111)$ surfaces. They first performed ab-initio calculations of the electronic structure in the framework of density functional theory and then used the results for dynamical simulations. Figure 3 shows a layered structure investigated in [160].

\section{SUMMARY}

One of the goals of computational nanoscience is to calculate physical and chemical properties from first principles. This requires the knowledge of the electronic structures of the materials system in question. The density-functional theory (DFT) makes a huge step towards this goal, by reducing a highly complex many-electron problem to that of noninteracting particles under the influence of an effective potential. The adiabatic approximation allows one to separate the ionic degrees of freedom from those of the electrons. However, in order to apply DFT in practice one has to resort to approximations for electron exchange and correlation such as the local-density approximation (LDA) or the generalized-gradient approximation (GGA). In this chapter, we have outlined some basic principles and some important examples of this approach, representing a very large and growing body of experimental and theoretical work on magnetic nanostructures such as clusters, nanowires, nanocontacts, and multilayers.

\section{References}

[1] P. Jena, S. N. Khanna, and B. K. Rao, Materials Science Forum 232, 1 (1996).

[2] B. Delley, D. E. Ellis, and A. J. Freeman, J. Magn. Magn. Mater. 30, 71 (1982).

[3] D. P. Pappas, A. P. Popov, A. N. Anisimov, B. V. Reddy, S. N. Khanna, Phys. Rev. Lett. 76, 4332 (1996)

[4] Primer in Density Functional Theory (Lecture Notes in Physics 620; Computational Materials Science. From Basic Principles to Material Properties; Lecture Notes in Phys. Vol. 642).

[5] O. K. Andersen, Phys. Rev. B 12, 3060 (1975)

[6] O. K. Andersen and O. Jepsen, Phys. Rev. Lett. 53, 2571 (1984).

[7] O. K. Andersen, Euro Phys. News 12, 1 (1981).

[8] H. L. Skriver, "The LMTO Method," Springer, Berlin 1984.

[9] A. R. Williams, J. Kubler, and C. D. Gelatt, Phys. Rev. B 19, 6094 (1979).

[10] C. Koenig, N. Stefanou, and J. M. Koch, Phys. Rev. B 33, 5307 (1986).

[11] O. Gunnarson, O. Jepsen, and O. K. Andersen, Phys. Rev. B 27, 7144 (1983).

[12] J. Harris, in "The Electronic Structure of Complex Systems," eds. W. Temmerman and P.
Phariseau, Plenum, New York 1984

[13] F. Casula and F. Herman, J Chem. Phys. 78, 858 (1983).

[14] M. Springborg and O. K. Andersen, J. Chem. Phys. 87, 7125 (1987).

[15] M. Springborg and R. O. Jones, Phys. Rev. Lett. 57, 1145 (1986).

[16] T. Fuziwara, J. Phys. F 16, 869 (1986).

[17] G. F. Fernando, B. R. Cooper, M. V. Ramana, H. Krakauer, and C. Q. Ma, Phys. Rev. Lett. 56, 2299 (1986).

[18] D. Glotzel, B. Segall, and O. K.Andersen, Solid State Commun. 36, 403 (1980).

[19] P. Blaha, K. Schwarz, and P. Sorantin, Computer Physics Communications 59, 399 (1990). See also wien $2 \mathrm{k}$ package.

[20] H. J. F. Jansen and A. J. Freeman, Phys. Rev. B 30, 561 (1984).

[21] M. Methfessel and M. van Schilfgaarde, Inter. J. of Modern Physics B. 7, 262 (1993).

[22] K. Koepernik and H. Eschrig, Phys. Rev. B 59, 1743 (1999).

[23] J. Wang and T. L. Beck, Journal of Chemical Physics 112, 9223 (2000).

[24] G. Kresse and J. Furthmuller, Phys. Rev. B 54, 11169 (1996).

[25] G.-M. Rignanese, X. Gonze, A. Pasquarello, "Ab-Initio Calculations of the Structural, Electronic and Dynamical Properties of High-k Dielectrics," ed. M. Houssa, Institute of Physics Publishing, 2004, p. 431.

[26] M. M. G. Alemany, M. Jain, L. Kronik, and J. R. Chelikowsky, Phys. Rev. B 69, 75101 (2004).

[27] R. Haydock, in "Solid State Physics: Advances in Research and Applications," eds H. Ehrenreich, F. Seitz, D. Turnbull, Academic, New York 1980, Vol. 35, p. 216.

[28] J. Gaspard and F. Cyrot-Lackmann, J. Phys C 6, 3077 (1973).

[29] W. A. Harrison, "Electronic Structure and Properties of Solids," Freeman, San Francisco 1980.

[30] J. C. Slater and G. F. Koster, Phys. Rev. 94, 1498 (1954).

[31] J. Fidler and T. Schreft, J. Phys. D 33, R135 (2000).

[32] U. von Barth and L. Hedin, J. Phys. C 5, 1629 (1972).

[33] O. Gunnarson, J. Phys. F 6, 587 (1976).

[34] J. F. Janak, Phys. Rev. B 16, 255 (1977).

[35] D. G. Pettifor, J. Magn. Magn. Mater. 15, 847 (1980)

[36] H. Takayama, K. P. Bohnen, and P. Fulde, Phys. Rev. B 14, 2287 (1976).

[37] J. G. Gay and R. Richter, Phys. Rev. Lett. 56, 2728 (1986).

[38] P. Bruno, Phys. Rev. B 39, 865 (1989).

[39] S. Pick and Dreyssee, Phys. Rev. B, 48, 13588 (1993).

[40] M. Cinal, D. M. Edwards, and J. Mathon, Phys. Rev. B 50, 3754 (1994).

[41] H. Victora and J. M. McLaren, Phys. Rev. B 47, 11583 (1993).

[42] J. Trygg, B. Johanson, O. Erikson, and J. M. Wills, Phys. Rev.Lett. 75, 2871 (1995).

[43] G. H. O. Daalderop, P. J. Kelly, and M. F. H. Schuurmans, Phys.Rev. B 41, 11919 (1990); 42, 7270 (1990). 
[44] C. Li, A. J. Freeman, H. J. F. Jansen, and C. L. Fu, Phys. Rev. B 42, 5433 (1990).

[45] D. S. Wang, R. Wu, and A. J. Freeman, Phys. Rev. Lett. 70, 869 (1993).

[46] A. R. Mackintosh and O. K. Andersen, in "Electrons at the Fermi Surface," ed. M. Sprinford, Cambridge University Press, Cambridge 1980, Chap. 53.

[47] V. Heine, in "Solid State Physics - Advances in Research and Applications," eds. H. Ehrenreich, D. Turnbull, and F. Seitz, Academic Press, New York 1980, Vol. 35, p. 114.

[48] A Aharoni, J. Magn. Magn. Mater. 197, 786 (1999).

[49] K. Moorjani and J. M. D. Coey, “Magnetic Glasses," Elsevier, Amsterdam 1984.

[50] M. Uhl and J. Kubler, Phys. Rev. Lett. 77, 334 (1996).

[51] A. I. Liechtenstein, M. I. Katsnelson, V. P. Antropov, and V. A. Gubanov, J. Magn. Magn. Mater. 67, 65 (1987).

[52] V. A. Gubanov, A. I. Liechtenstein, and A. V. Postnikov, "Magnetism and the Electronic Structure of Crystals," Springer, Berlin 1992.

[53] R. F. Sabirianov and S. S.Jaswal, Phys. Rev B 57, 7767 (1998).

[54] A. Kashyap, R. Skomski, R. F. Sabiryanov, S. S. Jaswal, and D. J. Sellmyer, IEEE Trans. Magn. 39, 2908 (2003).

[55] A. Kashyap, R. Skomski, A. K.Solanki, Y. F. Xu, and D.J. Sellmyer, J. Appl. Phys. 95, 7480 (2004).

[56] W. Hansen, J.P. Kottaus, and U. Merkt, Semiconductor Semimetals 35, 279 (1992).

[57] M. Kastner, Phys. Today, Jan 24 (1993).

[58] P. Ordejon, Comp. Mat. Sci. 12, 157 (1998).

[59] I. M. L. Billas, A. Chatelain, and W. A. de Heer, Science 265, 1682 (1994).

[60] S. E. Apsel, J. W. Emmert, J. Deng, and L. A. Bloomfield, Phys. Rev. Lett. 76, 1441 (1996).

[61] M. Respaud, M. Goiran, and J. M. Broto, Phys. Rev. B 59, R3934 (1999).

[62] K. Lee, J. Callaway, and S. Dhar, Phys. Rev. B 30,1724 (1985).

[63] C. Jamorski, A. Martinez, M. Castro, and D. R. Salahub, Phys. Rev. B 55, 10905 (1997).

[64] Y. Jinlong, F. Toigo, and W. Kelin, Phys. Rev. B 50, 7915 (1994).

[65] G. M. Pastor, "Atomic Clusters and Nanoparticles," NATO ASI Series, Les Houches Session LXXIII, eds. C. Guet, P. Hobza, F. Spiegelman, and F. David, Springer, Berlin 2001, p. 335 .

[66] V. Kumar and Y. Kawazoe, Phys. Rev. B 66, 144413 (1994).

[67] A. J. Cox, J. G. Louderback, and L. A. Bloomfield, Phys. Rev. Lett. 71, 923 (1993).

[68] A. J. Cox, J. G. Louderback, S. E. Apsel, and L. A. Bloomfield, Phys. Rev. B 49, 12295 (1994).

[69] B. V. Reddy, S. N. Khanna, and B. I. Dunlap, Phys. Rev. Lett. 70, 3323 (1993).

[70] G. Ganteför and W. Eberhardt, Phys. Rev. Lett. 76, 4975 (1996).

[71] G. M. Pastor, J. Dorantes-Dávila, and K. H. Bennemann, Phys. Rev. B 40, 7642 (1989).

[72] J. Dorantes-Dávila, H. Dreysse and G. M. Pator, Phys. Rev. B 46, 10432 (1992).

[73] F. Lopez-Urias, G. M. Pastor, and K. H. Bennemann, J. Appl. Phys. 87, 4909 (2000).
[74] B. Piveteau, M. C. Desjonquéres, A. M. Oles, and D. Spanjaard, Phys. Rev. B 53, 9251 (1996).

[75] J. L. Rodriguez-López, F. Aguilera-Granja, A. Vega, and J. A. Alonso, Eur. Phys. J. D 6, 235 (1999).

[76] R. Guirado-López, M. C. Desjonquéres, and D. Spanjaard, Phys. Rev. B 62, 13188 (2000).

[77] M. Jamet, M. Negrier, V. Dupuis, J. Tuaillon-Combes, P. Melinon, A. Perez, W. Wernsdorfer, B. Barbara, and B. Baguenard, J. Magn. Magn. Mater. 237, 293 (2001).

[78] M. Jamet, W. Wernsdorfer, C. Thirion, D. Mailly, V. Dupuis, P. Melinon, and A. Perez, Phys. Rev. Lett. 86, 4676 (2001).

[79] A. Thiaville, Phys. Rev. B 61, 12221 (2000).

[80] R. Dittrich, A. Thiaville, J. Miltat, and T. Schrefl, J. ofAppl. Phys. 93, 7891 (2003).

[81] R. F. Sabiryanov, M. I. Larsson, K. Cho, W. D. Nix, B. M. Clemens, Phys. Rev. B 67, 125412 (2003).

[82] P. Gambardella, S. Rusponi, M. Veronese, S. S. Dhesi, C. Grazioli, A. Dallmeyer, I. Cabria, R. Zeiler, P. H. Dederichs, K. Kern, C. Carbone, and H. Brune, Science 300, 1130 (2003).

[83] A. N Andriotis and M. Menon, Phys. Rev. Lett. 93, 026402/1 (2004).

[84] P. Lang, V. S. Stepanyuk, K. Wildberger, R. Zeiler, and P. H. Dederichs, Solid State Commun. 92, 755 (1994).

[85] K. Wildberger, V. S. Stepanyuk, P. Lang, R. Zeiler, and P. H. Dederichs, Phys. Rev. Lett. 75, 509 (1995).

[86] B. Nonas, K. Wildberger, R. Zeiler, and P. H. Dederichs, Phys. Rev. Lett. 80, 4574 (1998).

[87] N. A. Levanov, V. S. Stepanyuk, W. Hergert, D. I. Bazhanov, P. H. Dederichs, A. A. Katsnelson, and C. Massobrio, Phys. Rev. B 61, 2230 (2000).

[88] V. S. Stepanyuk and W. Hergert, Phys. Rev. B 62, 7542 (2000).

[89] I. Cabria, B. Nonas, R. Zeiler, and P. H. Dederichs, Phys. Rev. B 65, 054414 (2002).

[90] B. Lazarovits, L. Szunyogh, and P. Weinberger, Phys. Rev. B 65, 104441 (2002).

[91] Y. Qiang, R. Sabirianov, S. S. Jaswal, Y. Liu, H. Haberland, and D. J. Sellmyer, Phys. Rev. B 66, 064404 (1977).

[92] R. Robles, R. C. Longo, A. Vega, C. Rey, V. Stepanyuk, and L. J. Gallego, , Phys. Rev. B 66, 064410/1 (2002).

[93] E. F. Kneller and R. Hawig, IEEE Trans. Magn. 27, 3588 (1991).

[94] Z. Hao, L. Jing, J. P. Liu, Z. L. Wang, and Sun Shouheng, Nature 420, 395 (2002).

[95] H. Zeng, R. Sabirianov, O. Mryasov, K. Cho, and D. J. Sellmyer, Phys. Rev. B 66, 184425 (2002).

[96] Y. Kondo and K. Takayanag, Phys. Rev. Lett. 79, 3455 (1997).

[97] I. Lisiecki and A. Filankembo, Phys. Rev. B 61, 4968 (2000).

[98] J. L. Lin, D. Y. Petrovykh, A. Kirakosian, H. Rauscher, and F. J. Himpsel, Appl. Phys. Lett. 78, 829 (2001).

[99] B. H. Hong, S. C. Bae, C.-W. Lee, S. Jeong, K. S. Kirn, Science 294, 348 (2001).

[100] K. Kondo and K. Takayanagi, Science 289, 606 (2000). Y. Oshima, H. Koizumi, K. Mouri, H. Hirayama, and K. Takayanagi, Phys. Rev. B 65, 121401 (2002). 
[101] A. Fert and L. Piraux, J. Magn. Magn. Mater. 200, 338 (1999).

[102] R. Skomski, J. Phys.: Condens. Matter 15, R841 (2003).

[103] M. Wienert and A. J. Freeman, J. Magn. Magn. Mater. 38, 23 (1983).

[104] H. Jisang and R. Q. Wu, J. Appl. Phys. 93, 8764 (2003).

[105] A. Delin and E. Tosatti, J. Phys.: Condens. Matter 16, 8061 (2004).

[106] P. Gambardella, A. Dallmeyer, K. Maiti, M.C. Malagoli, S. Rusponi, P. Ohresser, W. Eberhardt, C. Carbone, and K. Kern, Phys. Rev. Lett. 93, 077203/1 (2004).

[107] D. Spisak and J. Hafner, Phys. Rev. B 65, 235405 (2002).

[108] Y. J. Jin, I. G. Kirn, and J. I. Lee, J. Korean Phys Soc 43, 1071 (2003).

[109] Y. J. Jin, I. G. Kirn, and J. I. Lee, Phys Stat Sol. (b) 241, 1431 (2004).

[110] P. Gambardella, A. Dallmeyer, K. Maiti, M. C. Malagoli, W. Eberhardt, K. Kern, and C. Carbone, Nature (London) 416, 301 (2002).

[111] B. Wang, X. Chen, G. Chen, G. Wang, and J. Zhao, Solid State Commun. 129, 25 (2004).

[112] A. K. Solanki, R. F. Sabiryanov, E. Y. Tsymbal, and S. S. Jaswal, J. Magn. Magn. Mater. 272-276, 1730 (2004).

[113] H. D. Chopra and S. Z. Hua, Phys. Rev. B 66, 020403(R) (2002).

[114] H. Wan, H. Cheng, and N. Garcia, arXiv:cond-mat/0207516.

[115] N. Garcia, M. Munoz, V. V. Osipov, E. V. Ponizovskaya, G. G. Qian, I. G. Saveliev, Y.-W. Zhao, J. Magn. Magn. Mater. 240, 92 (2002).

[116] N. Garcia, M. Munoz, and Y.-W. Zhao, Phys. Rev. Lett. 82, 2923 (1999).

[117] G. Tatara, Y.-W. Zhao, M. Munoz, and N. Garcia, Phys. Rev. Lett. 83, 2030 (1999).

[118] J. J. Versluijs, M. A. Bari, and J. M. D. Coey, Phys. Rev. Lett. 87, 026601 (2001).

[119] L. R. Tagirov, B. P. Vodopyanov, and K. B. Efetov, Phys. Rev. B 63, 104428 (2001).

[120] P. Bruno, Phys. Rev. Lett. 83, 2425 (1999); V. A Molyneux, V. V. Osipov, and E. V. Ponizovskaya, Phys. Rev. B 65, 184425 (2002).

[121] M. Y. Zhuravlev, E. Y. Tsymbal, S. S. Jaswal, A. V. Vedyayev, and B. Dieny, App. Phys. Lett. 83, 3534 (2003).

[122] J. D. Burton, A. Kashyap, M. Ye, Zhuravlev, R. Skomski, E. Y. Tsymbal, S. S. Jaswal, O. N. Mryasov, and R. W. Chantrell, App. Phys. Lett. 85, 251 (2004).

[123] J. C. Cuevas, A. Levy Yeyati, and A. Martín-Rodero, Phys. Rev. Lett. 80, 1066 (1998).

[124] A. Levy Yeyati, A. Martin-Rodero, and F. Flores, Phys. Rev. B 56,10369 (1997).

[125] M. Brandbyge, N. Kobayashi, and M. Tsukada, Phys. Rev. B 60, 17064 (1999).

[126] E. Scheer, N. Agrait, J. Carlos Cuevas, A. Levy Yeyati, B. Ludoloph, A. Martin-Rodero, G. Rubio Bollinger, J. M. van Ruitenbeek, and C. Urbina, Nature 394, 154 (1998).

[127] N. D. Lang, Phys. Rev. B 52, 5335 (1995).

[128] N. D. Lang, Phys. Rev. B 55, 9364 (1997).

[129] N. D. Lang and Ph. Avouris, Phys. Rev. Lett. 81, 3515 (1998).

[130] N. D. Lang and Ph. Avouris, Phys. Rev. Lett. 84, 358 (2000).

[131] N. Kobayashi, M. Brandbyge, and M. Tsukada, Jpn. J. Appl. Phys. 38, 336 (1999).

[132] N. Kobayashi, M. Aono, M. Tsukada, Phys. Rev. B 64, 121402 (2001).
[133] N. Kobayashi, M. Brandbyge, M. Tsukada, Phys. Rev. B 62, 8430 (2000).

[134] N. D. Lang, Phys. Rev. B 36, 8173 (1987); N. D. Lang, Phys. Rev. B 55, 9364 (1997).

[135] S. R. Bahn and K. W. Jacobsen, Phys. Rev. Lett. 87, 266101 (2001).

[136] H. Mehrez, A. Wlasenko, B. Larade, J. Taylor, P. Grütter, H. Guo, J. Wang, and C. Roland, Phys. Rev. B 65, 195419 (2002).

[137] M. Brandbyge, J. Mozos, P. Ordejon, J. Taylor, and K. Stokbro, Phys. Rev. B 65, 165401 (2002).

[138] J. Velev and W. H. Butler, Phys. Rev. B 69, 094425 (2004).

[139] E. G. Emberly and G. Kirczenow, Phys. Rev. B 60, 6028 (1999).

[140] M. N. Baibich, J. M. Broto, A. Pert, F. Nguyen Van Dau, and F. Petroff, P. Eitenne, G. Creuzet, A. Friederich, and J. Chazelas, Phys. Rev. Lett. 61, 2472 (1988).

[141] R. Allenspach, J. Magn. Magn. Mater. 129, 160 (1994).

[142] "Ultrathin Magnetic Structures I, II," eds. B. Heinrich and J. A. C. Bland, Springer, Berlin 1994.

[143] U. Gradmann, Ann. Phys. 7, 91 (1966); U. Gradmann, J. Magn. Magn. Mater. 54, 733 (1986).

[144] R. Allenspach, M. Stampanoni, and A. Bischoff, Phys. Rev. Lett. 65, 3344 (1990).

[145] B. Schuiz and K. Baberschke, Phys. Rev. B 50, 13467 (1994).

[146] F. Huang, M. T. Kief, G. J. Mankey, and R. F. Willis, Phys. Rev. B 49, 3962 (1994).

[147] G. Bochi, C. A. Ballentine, H. E. Inglefield, C. V. Thomson, R. C. O’Handley, Hans J. Hug, B. Stiefel, A. Moser, and H. J. Güntherodt, Phys. Rev. B 52, 7311 (1995).

[148] C. H. Lee, H. Hee, F. J. Lamelas, W. Vavra, C. Uher, and R. Clarke, Phys. Rev. B 42, 1066(1990).

[149] B. Heinrich, K. B. Urquardt, A. S. Arrott, J. F. Cochran, K. Martle, and S. T. Purcell, Phys. Rev. Lett. 59, 1756 (1987).

[150] Z. Q. Qiu, J. Pearson, and S. D. Bader, Phys. Rev. Lett. 70, 1006 (1993).

[151] C. Liu, E. R. Moog, and S. D. Bader, Phys. Rev. Lett. 60, 2422 (1988).

[152] D. P. Pappas, K. P. Kamper, and H. Hopster, Phys. Rev. Lett. 64, 3179 (1990).

[153] R. F. Sabiryanov and S. S. Jaswal, J. Magn. Magn. Mater. 177-181, 989 (1998); Phys. Rev. B 58, 12071 (1998)

[154] S. van Dijken, X. Jiang, and S. S. P. Parkin, App. Phys. Lett. 83, 951 (2003).

[155] E. Y. Tsymbal, O. N. Mryasov, and P. R. LeClair, J. Phys.: Cond. Matter 15, R109-R142 (2003).

[156] G. H. O. Daalderop, P. J. Kelly, and M. F. H. Schuurmans, Phys. Rev. B 50, 9989 (1994).

[157] R. Lorentz and J. Hafner, Phys. Rev. B 54, 15937 (1996).

[158] Le Bacq, O. Eriksson, and B. Johansson, Phys. Rev. B 65, 134430 (2002).

[159] B. Újfalussy, L. Szunyogh, P.Bruno, and P. Weinberger, Phys. Rev. B 77, 1805 (1996).

[160] V. S. Stepanyuk and W. Hergert, "Magnetism Structure and Interactions at the Atomic Scale," Lect. Notes Phys. 642, Springer, Berlin Heideberg 2004, 159-176. 